\title{
Molecular link between auxin and ROS-mediated polar growth
}

\author{
Silvina Mangano $o^{a, b, c, 1}$, Silvina Paola Denita-Juarez ${ }^{a, b, c, 1}$, Hee-Seung Choid ${ }^{d, 1}$ Eliana Marzol ${ }^{a, b, c, 1}$, Youra Hwang ${ }^{d}$, \\ Philippe Ranocha ${ }^{\mathrm{e}}$, Silvia Melina Velasquez ${ }^{\mathrm{a}, \mathrm{b}, c, 2}$, Cecilia Borassi ${ }^{\mathrm{a}, \mathrm{b}, \mathrm{c}}$, María Laura Barberinif, \\ Ariel Alejandro Aptekmann ${ }^{g}$, Jorge Prometeo Muschietti ${ }^{\mathrm{f}, \mathrm{h}}$, Alejandro Daniel Nadra ${ }^{\mathrm{g}}$, Christophe Dunand ${ }^{\mathrm{e}}$, \\ Hyung-Taeg Cho ${ }^{\mathrm{d}, 3}$, and José Manuel Estevez ${ }^{\mathrm{a}, \mathrm{b}, c, 3}$
}

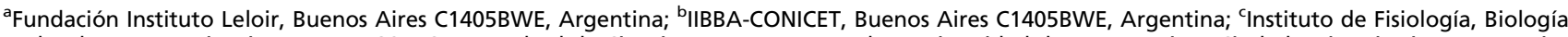
Molecular y Neurociencias, IFIByNE-CONICET, Facultad de Ciencias Exactas y Naturales, Universidad de Buenos Aires, Ciudad Universitaria, Buenos Aires C1428EGA, Argentina; ${ }^{d}$ Department of Biological Sciences, Seoul National University, Seoul 151-742, Korea; ${ }^{\text {e }}$ Laboratoire de Recherche en Sciences Végétales, Université de Toulouse, Université Paul Sabatier, UMR 5546, CNRS, F-31326 Castanet-Tolosan, France; ' Instituto de Investigaciones en Ingeniería Genética y Biología Molecular, Dr. Héctor Torres, INGEBI-CONICET, Buenos Aires 1428, Argentina; ${ }^{9}$ Departamento de Química Biológica,

IQUIBICEN-CONICET, Facultad de Ciencias Exactas y Naturales, Universidad de Buenos Aires, Ciudad Universitaria, Buenos Aires C1428EGA, Argentina; and 'hepartamento de Biodiversidad y Biología Experimental, Facultad de Ciencias Exactas y Naturales, Universidad de Buenos Aires, Ciudad Universitaria, Buenos Aires C1428EGA, Argentina
\end{abstract}

Edited by Natasha V. Raikhel, Center for Plant Cell Biology, Riverside, CA, and approved April 10, 2017 (received for review March 7, 2017)

Root hair polar growth is endogenously controlled by auxin and sustained by oscillating levels of reactive oxygen species (ROS). These cells extend several hundred-fold their original size toward signals important for plant survival. Although their final cell size is of fundamental importance, the molecular mechanisms that control it remain largely unknown. Here we show that ROS production is controlled by the transcription factor RSL4, which in turn is transcriptionally regulated by auxin through several auxin response factors (ARFs). In this manner, auxin controls ROS-mediated polar growth by activating RSL4, which then up-regulates the expression of genes encoding NADPH oxidases (also known as RESPIRATORY BURST OXIDASE HOMOLOG proteins) and class III peroxidases, which catalyze ROS production. Chemical or genetic interference with ROS balance or peroxidase activity affects root hair final cell size. Overall, our findings establish a molecular link between auxin and ROS-mediated polar root hair growth.

auxin | root hair growth | RSL4 | ROS | peroxidases

n Arabidopsis thaliana, root hair cells and non-hair cell layers differentiate from the epidermis in the meristematic zone of the root. Once root hair cell fate has been determined, root hairs protrude from the cell surface and represent up to $50 \%$ of the surface root area; this process is crucial for nutrient uptake and water absorption. Root hair growth is controlled by the interplay of several proteins, including the transcription factor (TF) ROOT HAIR DEFECTIVE 6 (RHD6), a class I RSL protein belonging to the basic helix-loop-helix (bHLH) family (1). The bHLH transcription factor RSL4 (ROOT HAIR DEFECTIVE SIXLIKE 4), which defines the final root hair length based on its level of expression (2), is a central growth regulator acting downstream of RHD6. Other TFs also contribute to the regulation of root hair growth $(3,4)$. Root hair cell elongation is modulated by a wide range of both environmental signals $(5,6)$ and endogenous hormones, such as ethylene and auxin $(2,5,7)$. However, the underlying mechanisms are unknown. The transcriptional auxin response is mediated by auxin binding to receptors of the TRANSPORT INHIBITOR RESPONSE 1/AUXIN SIGNALING F-BOX (TIR1/AFB) family and its coreceptor AUXIN/INDOLE 3-ACETIC ACID (Aux/IAA). This triggers the proteasome-dependent degradation of Aux/IAA, which results in the release of the auxin response factors (ARFs). The released ARFs bind to cis-auxin response elements (Aux-REs) in the promoters of early auxin response genes to trigger downstream responses (8). Gain-of-function mutants for several Aux/IAAs exhibit reduced root hair growth (9). On the other hand, a high degree of genetic redundancy is expected in root hairs because several ARFs are highly expressed in trichoblast cells [e.g., ARF7 and
ARF19 are the two most abundant ARFs (10)]. In agreement, the arf7arf19 double mutant did not show a reduction in root cell elongation (11), indicating that there are other ARFs acting downstream of auxin in growing root hair cells. Auxin needs to be sensed in situ in hair cells to trigger cell expansion, although no molecular connection has been proposed yet between ARFs and downstream TFs that control growth (SI Appendix, Fig. S1). Downstream auxin signaling is sustained by an oscillatory feedback loop composed of two main components, calcium ions $\left(\mathrm{Ca}^{2+}\right)$ and reactive oxygen species (ROS). High levels of cytoplasmic $\mathrm{Ca}^{2+}$ $\left({ }_{\text {cyt }} \mathrm{Ca}^{2+}\right)$ trigger ROS production by RBOHs (RESPIRATORY BURST OXIDASE HOMOLOG proteins), and high levels of ${ }_{\text {apo }}$ ROS activate unknown $\mathrm{Ca}^{2+}$-permeable channels that promote $\mathrm{Ca}^{2+}$ influx into the cytoplasm $(12,13)$. Plant RBOHs are plasma membrane-localized NADPH oxidases that produce apoplastic superoxide ion $\left(\mathrm{O}_{2}^{-}\right)$, which is mostly converted chemically or enzymatically into hydrogen peroxide $\left(\mathrm{H}_{2} \mathrm{O}_{2}\right)$. Arabidopsis $\mathrm{RBOHs}$

\section{Significance}

Tip-growing root hairs are an excellent model system for deciphering the molecular mechanism underlying reactive oxygen species (ROS)-mediated cell elongation. Root hairs are able to expand in response to external signals, increasing several hundred-fold their original size, which is important for survival of the plant. Although their final cell size is of fundamental importance, the molecular mechanisms that control it remain largely unknown. In this study, we propose a molecular mechanism that links the auxin-auxin response factors module to activation of RSL4, which directly targets genes encoding ROSproducing enzymes such as NADPH oxidases (or RESPIRATORY BURST OXIDASE HOMOLOG proteins) and secreted type III peroxidases. Activation of these genes impacts on apoplastic ROS homeostasis, thereby stimulating root hair cell elongation.

Author contributions: S.M., S.P.D.-J., H.-S.C., E.M., S.M.V., H.-T.C., and J.M.E. designed research; S.M., S.P.D.-J., H.-S.C., E.M., Y.H., P.R., S.M.V., C.B., M.L.B., C.D., and H.-T.C. performed research; S.M., S.P.D.-J., H.-S.C., E.M., Y.H., P.R., H.-T.C., and J.M.E. contributed new reagents/analytic tools; E.M., Y.H., P.R., C.B., M.L.B., A.A.A., J.P.M., A.D.N., C.D., H.-T.C., and J.M.E. analyzed data; and H.-T.C. and J.M.E. wrote the paper.

The authors declare no conflict of interest.

This article is a PNAS Direct Submission.

${ }^{1}$ S.M., S.P.D.-J., H.-S.C., and E.M. contributed equally to this work.

${ }^{2}$ Present address: Department of Applied Genetics and Cell Biology, University of Natural Resources and Life Sciences, 1190 Vienna, Austria.

${ }^{3}$ To whom correspondence may be addressed. Email: htcho@snu.ac.kr or jestevez@leloir. org.ar.

This article contains supporting information online at www.pnas.org/lookup/suppl/doi:10. 1073/pnas.1701536114/-/DCSupplemental. 
(AtRBOHA-J) are associated with diverse ROS-related growth responses (14-16); for instance, RBOHC promotes root hair budding $(17,18)$, and $\mathrm{RBOHH}, \mathrm{J}$ are required for proper pollen tube growth (19). In addition, the pool of apo ROS can be modulated by the activity of class III peroxidases (PERs) (20). The mechanism that links auxin to ROS-mediated cell growth is unclear (SI Appendix, Fig. S1). Here we propose a molecular mechanism in which endogenous auxin activates several ARFs to up-regulate the expression of RSL4, which controls ROS-mediated polar root hair growth by promoting the expression and activity of $\mathrm{RBOH}$ and PER proteins. On the other hand, we were not able to detect an auxin-mediated nontranscriptional fast response to trigger ROS production in root hair cells.

\section{Results and Discussion}

Several ARFs Mediate RSL4 Activation, Linking Auxin-Dependent Polar Growth to ROS Production in Root Hair Cells. To establish whether ROS production is linked to auxin-dependent polar growth, we analyzed root hair growth and ROS levels in the presence of exogenously supplied auxin (100 nM IAA). Auxin enhances root hair growth (Fig. $1 A$ and SI Appendix, Fig. S2A) and triggers a slightly higher ROS level in root hairs longer than $300 \mu \mathrm{m}$ (SI Appendix, Fig. S2B). Next, we monitored cytoplasmic $\mathrm{H}_{2} \mathrm{O}_{2}\left({ }_{\text {cyt }} \mathrm{H}_{2} \mathrm{O}_{2}\right)$ levels using a genetically encoded YFP-based $\mathrm{H}_{2} \mathrm{O}_{2}$ sensor, named HyPer, which reacts with $\mathrm{H}_{2} \mathrm{O}_{2}$ when external ${ }_{\text {apo }} \mathrm{H}_{2} \mathrm{O}_{2}$ is applied in the growing tip (SI Appendix, Fig. $\mathrm{S} 3 A$ ). $\mathrm{H}_{2} \mathrm{O}_{2}$ levels in auxin-treated roots maintain a moderate HyPer signal in root hairs longer than $700 \mu \mathrm{m}$, whereas there is no root hair development in nontreated ones that reach these cell lengths (SI Appendix, Fig. S3B). Hence, the maintenance of ${ }_{\text {cyt }}$ ROS levels is a reliable response to auxin in tip-growing root hairs. Auxin triggers polar root hair growth via an unknown mechanism involving RSL4 activation (5). First, we analyzed whether both RSL2 and RSL4 directly regulate ROS production to trigger root hair growth. The rsl2-1 rsl4-1 double mutant showed highly reduced ROS levels, whereas slightly higher ROS levels were detected in the RSL4-overexpressing line (RSL4 ${ }^{\mathrm{OE}}$ ) than in the wild type (WT) during late stages of root hair development ( $\geq 400 \mu \mathrm{m}$ in length) (Fig. $1 B$ and $C$ ). Therefore, both active RSL2 and RSL4 stimulate ROS production (Fig. $1 C$ and SI Appendix, Fig. S2D). We then tested whether auxin triggers ROS production in an RSL2/RSL4-dependent manner. Auxin partially stimulated root hair growth and ROS production in each of the rsl4-1 and rsl2-1 null mutants but failed in the rsl2-1 rsl4-1 double mutant (Fig. $1 A$ and $B$ and SI Appendix, Fig. S2 $C$ and $D$ ). Mutants for other reported TFs involved in root hair growth, such as mamyb (3) and hdg11 (4), were treated with auxin, but normal responses were observed, suggesting that these TFs are not involved in auxin activation (SI Appendix, Fig. S4). In addition, the $\mathrm{RSL}^{\mathrm{OE}}$ line was less sensitive to the RBOH inhibitor VAS2870 (VAS) with respect to cell growth-linked ROS production (SI Appendix, Fig. S5). Overall, these findings highlight the key role of both RSL2 and RSL4, although RSL4 has a more protagonist role as a main transcriptional activator of the ROS-related auxin response in root hairs.

Next, we tested whether ARFs regulate root hair growth by upregulating RSL4 expression and ROS production. Several ARFs, such as ARF5, ARF7, ARF8, and ARF19, are expressed in the meristematic and elongation zones of the root as well as in trichoblast cells and developing root hairs (SI Appendix, Fig. S6 $A$ and $B)$. Based on the lack of phenotype for the $\operatorname{arf7} \operatorname{arf19}$ mutant (11), all data suggest a high degree of functional redundancy between these ARFs possibly involved in root hair growth. To overcome this, we then generated lines overexpressing these ARFs under the control of the strong root hair-specific promoter EXPA7 (p7:ARF; SI Appendix, Fig. S6C). Root hair growth was enhanced in three overexpression lines tested, especially in the ARF5 line (Fig. $1 D$ and SI Appendix, Fig. S6C). Furthermore, RSL4 expression was elevated

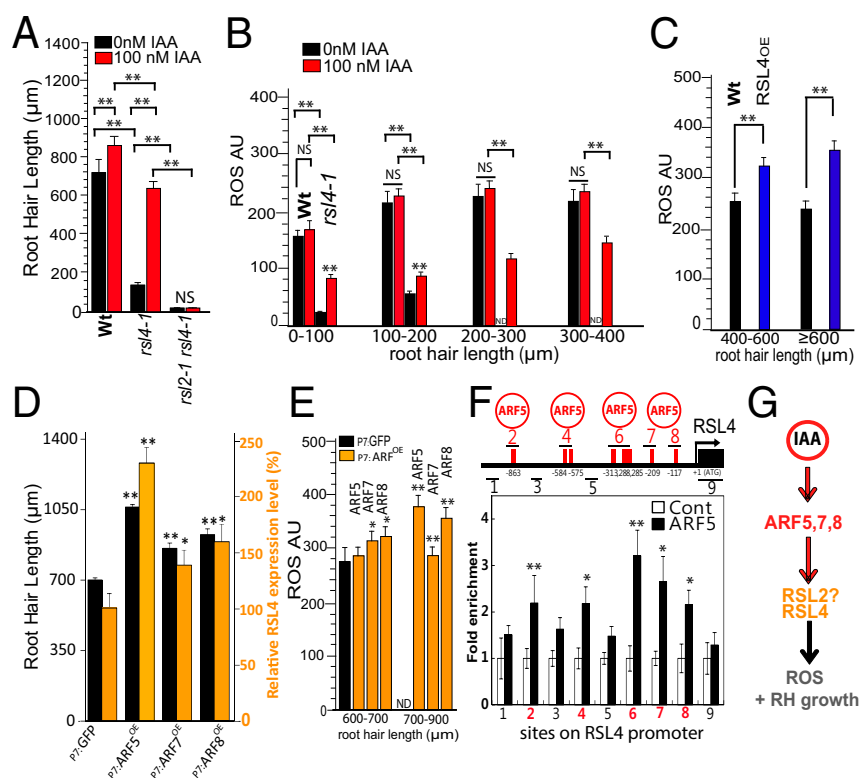

Fig. 1. ARF-mediated RSL4 activation links auxin-dependent polar root hair growth to ROS production. $(A)$ Root hair length (mean \pm SD) in WT Col-0, rs/4-1, and rs/2-1 rs/4-2 treated with $100 \mathrm{nM}$ IAA. (B) Effect of auxin (100 nM IAA) mediated by RSL4 on ROS production. ROS signal analysis with IAA treatment of WT Col- 0 and the $r$ /4-1 mutant in the early stages of root hair development. AU, fluorescent arbitrary units. (C) ROS in WT Col-0 and the RSL4 overexpressor in later stages of root hair growth. (D) Effect of EXP7 (EXPANSIN 7)-driven ARF5 ( 7 7:ARF5 ${ }^{\mathrm{OE}}$ ), ARF7 ( $\mathrm{P7}$ : $\mathrm{ARF7}{ }^{\mathrm{OE}}$ ), and ARF8 (P7:ARF8 ${ }^{\mathrm{OE}}$ ) overexpression on polar root hair growth (black bars) and RSL4 expression (orange bars) (mean \pm SD). (E) ROS in EXP7-GFP/WT Col-0 (P7:GFP as control) and the EXP7-driven ARF5,7,8 (P7:ARF5,7,8 ${ }^{\mathrm{OE}}$ ) overexpressor in the later stages of root hair development. $(F)$ ChIP analysis showing ARF5 binding to auxin response elements on the RSL4 promoter region. The RSL4 promoter region (pRSL4) and the relative positions of Aux REs (red bars) and ChIP-PCR regions (lines 1 to 9) are indicated. (Bottom) The enrichment fold of ARF5-GFP in ChIP-PCR of each region shown. Cont, control, empty vector line; ARF5, pMDC7:ARF5-GFP, dexamethasone-inducible ARF5-GFP line. The values are relative to each Cont value and significantly different $\left(* * P<0.001,{ }^{*} P<0.005\right.$; $t$ test) from each Cont value. $(G)$ Proposed events from the IAA signal mediated by ARF5 activation of $R S L 4$ expression to ROS-linked root hair (RH) growth. $(A-E)$ One-way ANOVA; $* * P<0.001,{ }^{*} P<$ 0.01 . ND, not detected; NS, not significantly different. Error bars indicate \pm SD of biological replicates.

(Fig. 1D) and high ROS levels were maintained in the root hair cells of these lines during late developmental stages (Fig. $1 E$ ). Given that the region upstream of RSL4 contains eight ARF response elements (Fig. $1 F$ and SI Appendix, Table S2), we speculated that these ARFs would be able to bind to the RSL4 promoter and trigger its expression. To test this possibility, we carried out chromatin immunoprecipitation (ChIP) analyses (Fig. $1 F$ ) using an estradiolinducible version of ARF5-GFP because it was the most efficient ARF tested to up-regulate RSL4 expression. ChIP of ARF5 showed that the fold-enrichment levels are significantly higher in the Aux-RE regions than in the control (Fig. $1 F$ ). These data indicate that ARF5 binds to Aux-REs in the RSL4 promoter region and positively regulates its expression. It is highly possible that ARF7 and ARF8 also bind and regulate RSL4 expression. Previously, it was shown that the Aux/IAA14 slr1-1 resistant mutant lacked root hairs (9), suggesting a strong inhibition of these ARFs. To test whether a single ARF is able to overcome this inhibition, we selected ARF19 because it is one of the ARFs most highly expressed in root hair cells (10). High levels of ARF19 (ARF19 ${ }^{\circ}$; overexpression) were transgenically expressed in the Aux/IAA14 slr1-1 root hair-less background. $\mathrm{ARF} 19^{\mathrm{OE}}$ was able to completely rescue the slr1-1 root hair phenotype and normalize ROS levels (SI Appendix, Fig. S7 $A$ and 
$B)$. As expected, ARF19 nuclear expression is highly responsive to auxin and is localized in root epidermal cells close to the root hair development zone (SI Appendix, Figs. S6B and S7C). Together, these results and previous reports (9-11) suggest that high auxin levels in trichoblast cells would release several ARFs (e.g., ARF5, ARF7, ARF8, and ARF19) from its repressors Aux/IAAs, to directly control RSL4 expression (and possibly RSL2), triggering ROS-mediated root hair elongation (Fig. $1 F$ ). Further experiments are required to establish how these ARFs are regulated to act in a coherent manner to trigger the transcriptional response during cell growth.

RSL4-Regulated RBOHC, J as Well as RBOHH Drive ROS-Mediated Root Hair Growth. Besides RBOHC, two other RBOH groups exist: One group clusters RBOHC and is composed of RBOHE,F (SI Appendix, Fig. S8A), and the other was previously implicated in polar growth of pollen tubes (19) and is composed of RBOHH,J. To identify which of these RBOHs also contribute to ROS-linked tip growth, we isolated rboh mutants (SI Appendix, Fig. S8B and Table S1) and screened them for abnormal cell expansion linked to deficient ROS production (Fig. $2 A$ and $B$ ). Only rbohc-1 and $c-2$ showed short root hairs, whereas rbohh-1 and $h-3$ developed slightly less elongated cells (SI Appendix, Fig. S8 $C$ and $D$ ). When double rboh mutants were analyzed, only rbohh,j and mutants containing $r b o h c$ showed a clear root hair growth reduction of up to $40 \%$ (Fig. $2 A$ ). In agreement with this, rbohc mutants showed highly reduced ROS levels, whereas double rbohh,j mutants presented moderate to low ROS levels (Fig. $2 B$ and SI Appendix, Fig. S8 $E$ and $F$ ). We then used the $\mathrm{HyPer} \mathrm{H}_{2} \mathrm{O}_{2}$ sensor to test whether the $\mathrm{RBOH}$ inhibitor VAS could limit the level of ${ }_{\mathrm{cyt}} \mathrm{H}_{2} \mathrm{O}_{2}$ in the growing root hair tip. The level of ${ }_{\mathrm{cyt}} \mathrm{H}_{2} \mathrm{O}_{2}$ was significantly diminished after VAS treatment (Fig. $2 \mathrm{C}$ ), and ${ }_{\text {cyt }} \mathrm{H}_{2} \mathrm{O}_{2}$ signatures were drastically modified in rbohc-1 root hairs (Fig. 2D). Exogenous $\mathrm{H}_{2} \mathrm{O}_{2}$ treatment failed to rescue the noxc-1 root hair growth defect (SI Appendix, Fig. S9A), implying that a complex and finetuned ROS regulation would be required to trigger growth. Together, these data suggest that although RBOHC is the main $\mathrm{RBOH}$ involved in ROS-mediated root hair growth, as reported previously $(12,17), \mathrm{RBOHH}, \mathrm{J}$ are also important ROS-producing enzymes in this process.

Based on an analysis of RSL4-regulated genes (5), a putative RSL4 response element (RSL4-RE) with the sequence $\mathrm{TN}_{6} \mathrm{CA}[\mathrm{CT}] \mathrm{G}[\mathrm{TA}]$ was identified that is highly similar to the root hair cis-element (RHE) $\mathrm{TN}_{5-6} \mathrm{CACG}$ [TA]. The promoter regions of the $R B O H C$, $H$, and $J$ genes include several RHEs, suggesting that these three RBOHs could be direct targets of the RSL4 TF (SI Appendix, Table S2). Using ChIP analysis, we examined whether RSL4-GFP could bind to the promoter regions of RBOHC,H,J in vivo. Except for the RBOHH gene, RSL4 bound to at least one RHE region in the RBOHC and RBOHJ promoters (Fig. $2 E$ ). This result suggests that RSL4 directly regulates these two ROS-related genes to generate the ROS required for tip growth.

Why are three $\mathrm{RBOH}$ proteins needed to trigger polar growth in a single cell? The simplest hypothesis is that these RBOHs act sequentially. Low levels of ROS were detected when the root hair first emerged from the root, but the ROS signal was up to twofold greater in slightly longer WT Col-0 root hairs (of 200 to $250 \mu \mathrm{m}$ in length) (SI Appendix, Fig. S9 $D$ and $E$ ). At a comparable stage, root hairs of the rbohc-1 mutant had very low levels of cytROS and those of the rbohh,j double mutant had only slightly lower ROS levels than did those of WT Col-0. In later growth stages, cyt ROS levels were drastically reduced in the double rbohh,j mutant. This suggests that RBOHC is the first $\mathrm{RBOH}$ to produce ROS, and that RBOHC functionally overlaps with RBOHH,J. Finally, $\mathrm{RBOHH}, \mathrm{J}$ produced ROS that was linked to root hair tip elongation at later developmental stages (>200 $\mu \mathrm{m}$ in length) $(S I$ Appendix, Fig. S9E). Next, we investigated whether ROS is required for root hair initiation by subjecting WT Col-0 roots to
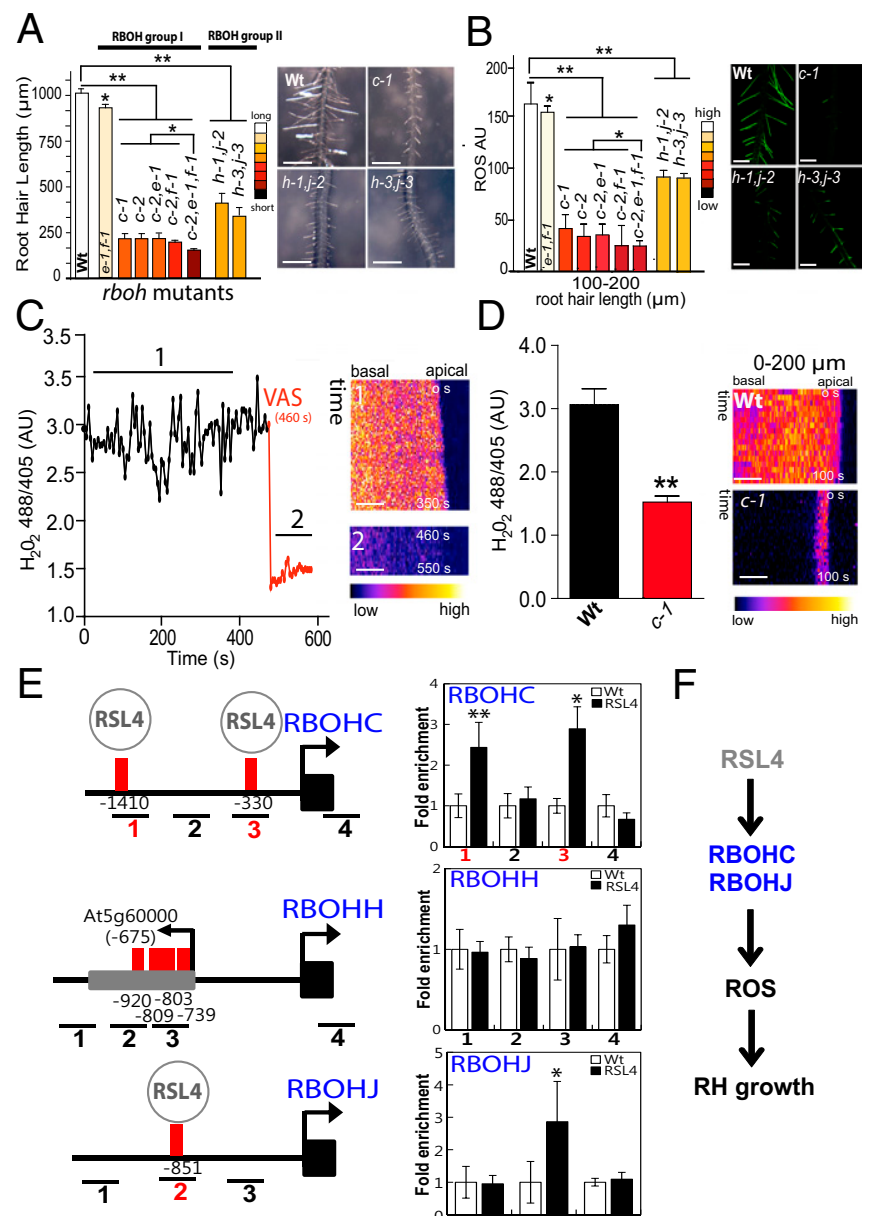

F
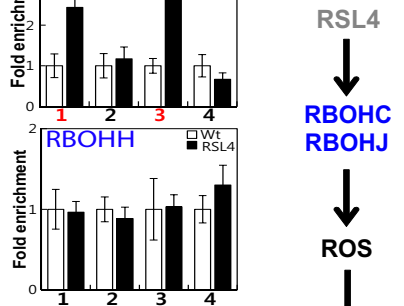

RBOHJ
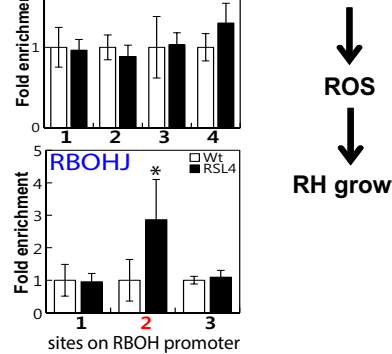

RH growth

Fig. 2. RSL4-regulated $R B O H C$, $J$, as well as $H$ drive ROS-mediated root hair growth. ( $A$, Right) Root hair phenotype in WT Col-0 and rboh mutants. (Scale bars, $600 \mu \mathrm{m}$.) (Left) Root hair length (mean $\pm \mathrm{SD} ; n=30$ roots) in rboh mutants. (B) Total ROS generated by oxidation of $\mathrm{H}_{2}$ DCF-DA in early stages of root hair development in rboh mutants. (Right) Total ROS $\mathrm{H}_{2}$ DCF-DA staining in roots of WT Col-0 and rboh mutants. (Scale bars, $600 \mu \mathrm{m}$.) (C) ${ }_{\mathrm{cyt}} \mathrm{H}_{2} \mathrm{O}_{2}$ levels in VAS-treated WT Col-0 root hairs expressing the HyPer sensor. cyt $\mathrm{H}_{2} \mathrm{O}_{2}$ levels are based on the ratio $488 / 405 \mathrm{~nm}$ of HyPer biosensor at the root hair tip over $600 \mathrm{~s}$. (Right) Selected kymographs resulting from this analysis only for root hairs of $>200 \mu \mathrm{m}$ in length. (Scale bars, $5 \mu \mathrm{m}$.) $(D){ }_{\text {cyt }} \mathrm{H}_{2} \mathrm{O}_{2}$ average levels in rbohc- 1 and WT Col- 0 root hairs expressing HyPer. Average ${ }_{\text {cyt }} \mathrm{H}_{2} \mathrm{O}_{2}$ levels are based on the $488 / 405 \mathrm{~nm}$ ratio of HyPer at the root hair tip in root hairs of 0 to $200 \mu \mathrm{m}$ in length. (Right) Selected kymographs. (Scale bars, $5 \mu \mathrm{m}$.) (E) Promoter regions of ROS-related genes $R B O H C, H$, and $J$ as targets of the RSL4 response element (red bars) and ChIP-PCR regions (lines 1 to 4). Neighboring gene exons are indicated as a gray box. Enrichment fold of RSL4GFP in ChIP-PCR for each region shown. RSL4, pRSL4:RSL4-GFP. Error bars indicate \pm SD of biological replicates $(n=2)$. The values are relative to each Cont value. Significantly different $(* * P<0.01, * P<0.05 ; t$ test) from the Cont value. $(F)$ Proposed events from RSL4 activation of RBOHC,J to ROS-mediated polar root hair growth. $\left(A, B\right.$, and $D$ ) One-way ANOVA; ${ }^{*} P<0.001,{ }^{*} P<0.01$. Error bars indicate \pm SD of biological replicates.

VAS. The concentration of VAS needed to inhibit root hair growth by half $\left(\mathrm{IC}_{50}\right)$ was $7.5 \mu \mathrm{M}$ (SI Appendix, Fig. S9B), which is similar to ROS VAS $\operatorname{IC}_{50}(\sim 7.0 \mu \mathrm{M})$ (SI Appendix, Fig. S9C). The rbohc-1 mutant (12) showed very short root hairs up to $150 \mu \mathrm{m}$ in length (SI Appendix, Fig. S9B) but still produced $\sim 30 \%$ of ROS compared with WT root hairs (Fig. $2 D$ and SI Appendix, Fig. $\mathrm{S} 9 \mathrm{C}$ ), indicating the presence of residual ROS production (17). Because ROS homeostasis could be modified by the activity of PER, we treated WT and rbohc-1 mutant roots with the PER 
inhibitor SHAM (salicylhydroxamic acid) (21) at concentrations of up to $100 \mu \mathrm{M}$. SHAM treatment resulted in a significant reduction in total ROS in rbohc-1 in comparison with nontreated rbohc-1, although root hair initiation was not abolished (SI Appendix, Fig. S10). In an analysis using HyPer, $100 \mu \mathrm{M}$ SHAM completely abolished the $\mathrm{H}_{2} \mathrm{O}_{2}$ signal in the whole root (SI Appendix, Fig. S11), confirming that PERs also contribute to ROS production. This implies that, at least in $A$. thaliana, initial development of the root hair tip is not affected by highly reduced ROS levels.

RSL4-Regulated PERs Affect ROS Homeostasis and Polar Root Hair Growth. Next, coexpression analysis revealed that RSL4 is highly coregulated at the transcriptional level with several PER genes, most of which exhibit root hair expression (SI Appendix, Fig. S12 $A$ and $B$ ) (5). We then analyzed the corresponding T-DNA per mutants (SI Appendix, Fig. S12C and Table S1). The single mutant per1-2 and double mutant per44-2,73-3 (all null mutants except for per1-2 knockdown; SI Appendix, Fig. S12D) showed mild phenotypes (short root hairs), with up to $\sim 30 \%$ reductions in ROS levels (Fig. $3 A$ and $B$ ). This suggests that there is genetic redundancy between these PERs in the regulation of ROS homeostasis. The levels of ${ }_{\mathrm{cyt}} \mathrm{H}_{2} \mathrm{O}_{2}$, as visualized with HyPer, were reduced in root hairs treated with $75 \mu \mathrm{M}$ SHAM (Fig. $3 C$ ), and this result was confirmed using a $2^{\prime}$, $7^{\prime}$-dichlorodihydrofluorescein diacetate $\left(\mathrm{H}_{2} \mathrm{DCF}-\mathrm{DA}\right)$ probe $(\mathrm{SI}$ Appendix, Fig. S10B). In addition, peroxidase activity was significantly lower in SHAM-treated roots as well as in per mutants and higher in PER44 overexpressor (PER44 ${ }^{\mathrm{OE}}$ ) than in WT nontreated ones (Fig. 3D), suggesting a direct link between peroxidase activity and ROS homeostasis. To explore the transcriptional regulation of these PERs in ROS-mediated root hair growth, we analyzed the regulatory region of the genes encoding these PERs. We identified several putative RHE motifs (SI Appendix, Table S3), suggesting that these genes could be direct targets of RSL4, as suggested by the coexpression analysis ( $S I$ Appendix, Fig. S12A). ChIP assays revealed that RSL4 binds to the RHE of four PERs, PER1, 44, 60, and 73 (Fig. 3E). In agreement with this, much higher and lower levels of PERI and PER73 were detected in RSL4 ${ }^{\mathrm{OE}} / \mathrm{WT}$ and $\mathrm{WT} /$ rsl4- 1 lines, respectively (5), suggesting that RSL4 promotes the expression of at least these two PERs. At last, higher peroxidase activity was found in ${ }_{\mathrm{P} 7:} \mathrm{ARF} 5$ and $_{\mathrm{P} 7} \mathrm{ARF}$ 8 lines (where RSL4 expression is up-regulated; Fig. $1 D$ ) and much lower activity was detected in rsl4-1, which lacks RSL4 (Fig. 3F), confirming that RSL4 not only directs $P E R$ expression at the transcriptional level but also impacts on the PER activity (Fig. 3G).

We then examined whether auxin treatment could compensate for the lack of RBOH and PER proteins (Fig. $4 A$ and $B$ ). Auxin treatment partially rescued the reduced length and ROS levels of the rbohc-1 mutant, resulting in $\sim 30 \%$ increases in both, and almost fully rescued the defects in the rbohh,j and per44-2,73-3 mutants (Fig. $4 A$ and $B$ ). When $15 \mu \mathrm{M}$ VAS was added along with the auxin (IAA), the rbohc-1 mutant exhibited intermediate root hair growth and partial recovery of ROS levels (SI Appendix, Fig. S13 $A$ and $B$ ), suggesting other sources of ROS. When SHAM was added in addition to VAS (SHAM+VAS) in the presence of auxin, a low recovery of root hair growth and ROS levels was detected in rbohc-1 and WT root hairs (SI Appendix, Fig. S13 C and $D$ ). Together, these findings confirm that auxin requires both RBOH-derived ROS and ROS produced by PER to modulate polar growth (SI Appendix, Fig. S13E). The small although detectable recovery in ROS and cell growth after auxin treatment in root hair cells (in rbohc-1 as well as in WT) in the presence of both inhibitors, VAS and SHAM (SI Appendix, Fig. S13 $C$ and D), would possibly suggest the existence of an unknown source of ROS coming from other apoplastic ROS-producing enzymes (e.g., oxalate oxidase, diamine oxidase, lipoxigenases, etc.) also regulated

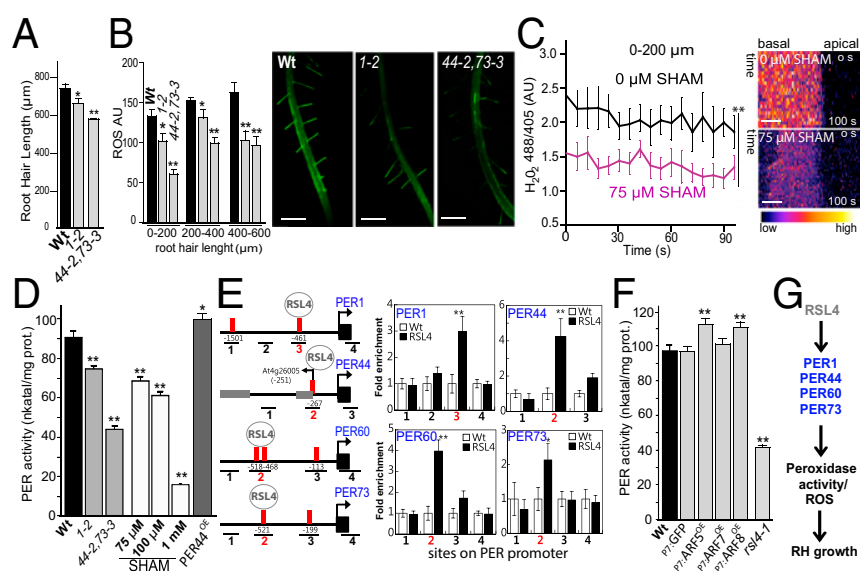

Fig. 3. RSL4-regulated PERs affect ROS homeostasis and polar root hair growth. (A) Root hair length (mean \pm SD; $n=30$ roots) in the single mutant per1-2 and double mutant per44-2,73-3. (B) ROS in root hairs of the single mutant per1-2 and double mutant per44-2,73-3. (Right) ROS $\mathrm{H}_{2}$ DCF-DA staining in roots of WT Col- 0 and per mutants. (Scale bars, $600 \mu \mathrm{m}$.) (C) Average ${ }_{c y t} \mathrm{H}_{2} \mathrm{O}_{2}$ levels in SHAM-treated and nontreated WT Col-0 root hairs expressing the HyPer sensor $(n=10)$. Average ${ }_{\text {cyt }} \mathrm{H}_{2} \mathrm{O}_{2}$ levels are based on the $488 / 405 \mathrm{~nm}$ ratio of HyPer at the root hair tip over $100 \mathrm{~s}$. One-way ANOVA; ${ }^{* * P}<0.001$ (Right) Selected kymographs. (Scale bars, $5 \mu \mathrm{m}$.) (D) Peroxidase activity in the roots of WT, per1-2, per44-2, and per73-3 simple mutants, per44-2,73-3 double mutant, and wild-type root extracts with increasing concentrations of SHAM. Enzyme activity (expressed as $n$ katal/mg protein) was determined by a guaiacol oxidation-based assay. Values are the mean of three replicates \pm SD. (E) Promoter regions of ROS-related genes PER1,44,60,73 showing the RSL4 response elements (red bars) and ChIP-PCR regions (lines 1 to 4). Positions are relative to the start codon. Neighboring gene exons are indicated as gray boxes. Enrichment fold of RSL4-GFP in ChIP-PCR of each region is shown. RSL4 pRSL4:GFP. Error bars indicate \pm SD of biological replicates $(n=2)$. The values are relative to each WT value and significantly different $(* * P<0.01, * P<0.05$; $t$ test). $(F)$ Peroxidase activity in the roots of WT, EXP7-driven GFP (as control), ARF5 ( mutant. Enzyme activity (expressed as $n \mathrm{katal} / \mathrm{mg}$ protein) was determined by a guaiacol oxidation-based assay. Values are the mean of three replicates \pm SD (G) Proposed events from RSL4 activation of PER1,44,60,73 to ROS-linked root hair growth. $(A, B, D$, and $F$ ) One-way ANOVA, $* * P<0.001, * P<0.01$. Error bars indicate \pm SD of biological replicates.

by auxin-RSL4 (and possibly RSL2) (SI Appendix, Fig. S13E), which will require further investigation.

Finally, we tested whether auxin is able to trigger a rapid and nontranscriptional response impacting on ROS production in the root hair tip (SI Appendix, Fig. S14). We applied IAA in growing root hairs that express the HyPer sensor, and no significant changes were detected before or after hormone treatment (SI Appendix, Fig. S14 $A$ and $B$ ). A similar result was obtained when ROS levels were measured with the dye $\mathrm{H}_{2}$ DCF-DA (SI Appendix, Fig. S14C). Overall, our results suggest that the main auxin downstream targets are RSL4 (and possibly RSL2) and the transcriptional activation of genes related to ROS production and involved in polar growth (SI Appendix, Fig. S13E). We cannot exclude other direct nontranscriptionally activated targets involved in the auxin response in root hair cells, but we fail to detect changes in $\mathrm{H}_{2} \mathrm{O}_{2} / \mathrm{ROS}$ upon auxin stimulation in our experimental conditions.

\section{Conclusions}

A root hair is a single-cell protrusion able to extend in response to external signals, increasing several hundred-fold its original size, which is important for survival of the plant. Final root hair size has vital physiological implications, determining the surface area/volume ratio of the whole roots exposed to nutrient pools, thereby likely impacting nutrient uptake rates. Although the final hair size is of fundamental importance, the molecular mechanisms that 
A

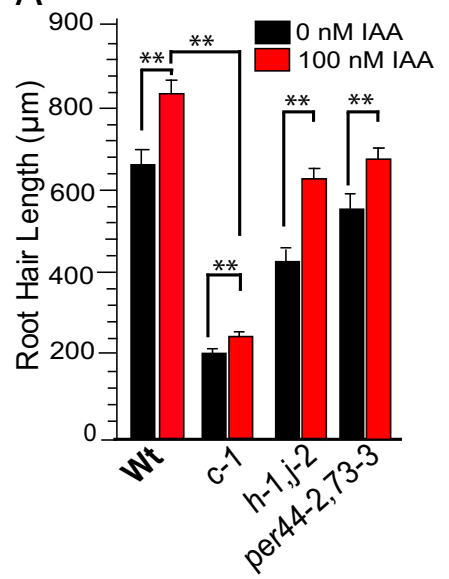

B
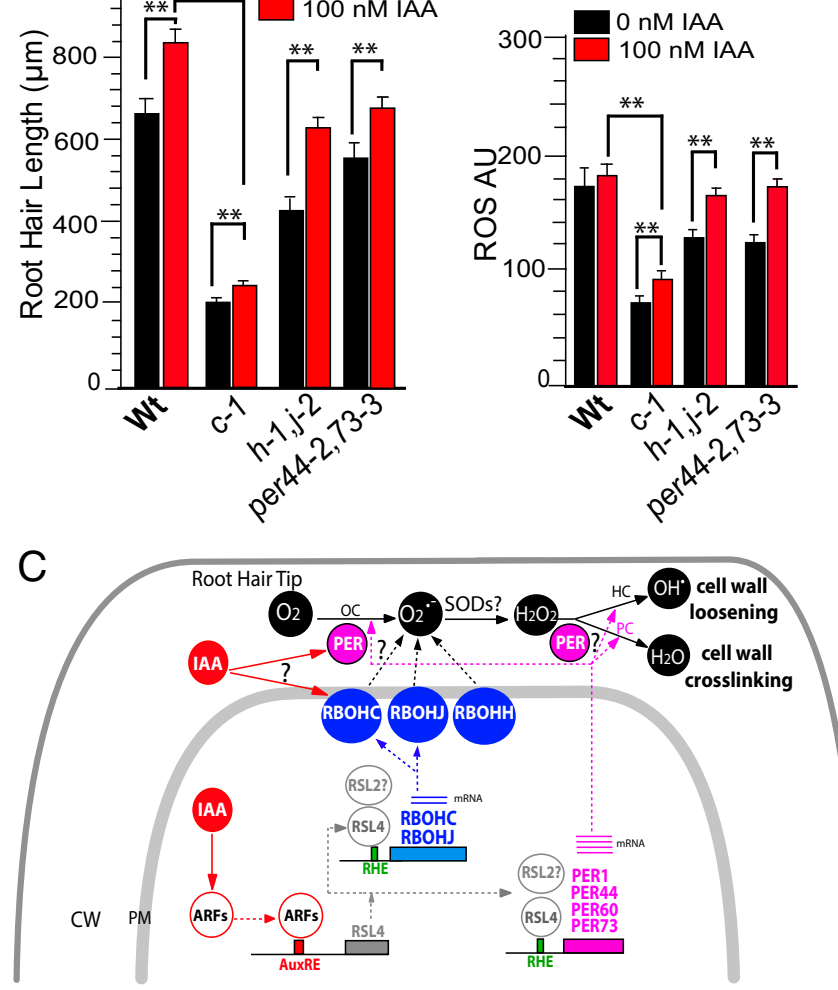

Fig. 4. Auxin-stimulated polar growth requires apoplastic ROS. (A) Root hair length (mean \pm SD; $n=30$ roots) in WT Col- 0 and $r b o h$ and per mutants treated or not with $100 \mathrm{nM}$ IAA. (B) ROS signal in WT and rboh mutants treated or not with $100 \mathrm{nM}$ IAA. Root hairs of 0 to $200 \mu \mathrm{m}$ in length were analyzed. (C) Model of auxin/ARF5/RSL4 regulation of ROS-mediated polar root hair growth. The bHLH transcription factor RSL4 is transcriptionally activated by auxin (IAA) and its expression is directly regulated by severa ARFs (e.g., ARF5,7,8,19). Through RSL4 (and possibly RSL2), auxin activates the expression of two RBOHs (RBOHC,J) and four PERs (PER1,44,60,73) that together regulate ROS homeostasis in the apoplast (in combination with $\mathrm{RBOHH}$ ). PERs in the presence of apoROS would control cell-wall loosening (in the peroxidative cycle; PC) and cross-linking reactions (in the hydroxylic cycle; $\mathrm{HC}$ ) that impact on polar growth. PERs also contribute to the superoxide radical pool by oxidizing singlet oxygen (in the oxidative cycle; $O C$ ). Solid lines indicate activation or chemical conversion; dashed lines indicate protein translation and targeting or contribution. CW, cell wall; PM, plasma membrane. Reactive oxygen species: hydroxyl radical, $\bullet \mathrm{OH}^{\circ}$ superoxide ion, $\mathrm{O}_{2} \bullet-$ singlet oxygen, $\mathrm{O}_{2}$; superoxide dismutases, SODs. ? indicates unidentified PER catalyzing the indicated reaction. $(A$ and $B$ ) One-way ANOVA; $* * P<0.001$. Error bars indicate \pm SD.

control it remain largely unknown. Here we propose a mechanism that explains how root hair tip growth works under auxin regulation of ROS homeostasis by activating several ARFs and subsequently up-regulating the expression of the bHLH TF RSL4 (and possibly RSL2). RSL2/RSL4-dependent ROS production is catalyzed by $\mathrm{RBOH}$ and PER enzymes, which facilitates polar root hair growth and defines its final cell size (Fig. 4C). Auxin is a prominent signal in trichoblast cells, and is essential for proper polar root hair growth. Auxin-ARF activation of the bHLH TF regulates several other plant developmental processes $(22,23)$. Here we have identified that auxin activates RSL4 via several ARFs (ARF5,7,8), which bind (at least ARF5) to several Aux-REs (8) in the RSL4 regulatory region. In addition, high levels of ARF19 are able to overcome a resistant Aux/IAA14, slr1-1, to trigger ROS-mediated polar growth. Recently, two dominant mutated versions of Aux/IAA7 were found to completely suppress root hair growth by down-regulating RSL4 expression (24). Based on these results, we hypothesize that IAA7-IAA14 directly repress several ARFs (ARF5,7,8,19) to control RSL4 (and RSL2) expression. Based on the mutant analysis, RSL2 would act together with RSL4 to trigger ROS responses when stimulated with auxin. Once RSL4 is active, it will trigger RSL2 expression $(2,5)$, and both are able to control ROS homeostasis during polar root hair growth by directly regulating the expression of $R B O H \mathrm{~s}$ and $P E R \mathrm{~s}$. Different types of ROS affect cell growth by modulating the balance between cell proliferation and cell elongation in the root meristem $(25,26)$ by an unknown molecular mechanism. In this study, we report that, under auxin induction, RSL2/RSL4 triggers ROS-mediated cell elongation. ChIP analysis revealed that RSL4 directly regulates the expression of two $\mathrm{RBOHs}$ as well as four PERs. However, out of 34 putative direct targets of RSL4 reported (27), only a few (e.g., PER7) were found to be associated with ROS homeostasis. In another recent report, RSL4 was shown to bind to RHE and trigger (directly or indirectly) the expression of the smallest subset of 124 genes involved in ROS homeostasis, cell-wall synthesis and remodeling, metabolism, and signaling that are necessary and sufficient to trigger root hair growth (28). Still unknown are the RSL2 targets and how RSL2 acts in a cooperative way with RSL4. In addition, the transcriptional mediator subunit (MED) MED15/PHYTOCHROME AND FLOWERING TIME 1 affects ROS levels by triggering transcriptional changes in root hair cells (29), probably via a mechanism that does not involve RSL4. Alternatively, ROS homeostasis may be influenced by other factors, independent of the RSL4 transcriptional program, that also modulate $\mathrm{RBOH}$ and PER activities, such as $\mathrm{Ca}^{2+}$ binding and posttranslational modifications $(18,30)$. Overall, here we have established a key role of RSL4 as one transcriptional activator of the ROS-related auxin response in root hairs together with RSL2, but we cannot discard that other TFs could also act downstream of auxin and independent of RSL2/RSL4 to trigger ROS production in root hair cells (SI Appendix, Fig. S13E).

Here we identified two RBOHs (RBOHH and J) together with the previously reported RBOHC (12) and four PERs (PER1, 44, 60, and 73) that are involved in ROS-mediated polar root hair growth in Arabidopsis (Fig. 4C). In agreement with our findings, an RBOH named RTH5 (root hair-less 5) from maize with high sequence similarity to AtRBOHH,J was also linked to root hair cell expansion (31). Several of the PERs identified as contributing to ROS-mediated root hair growth were previously found to modulate cell growth but were not shown to be involved in ROS homeostasis $(27,32)$. Exogenously supplied $\mathrm{H}_{2} \mathrm{O}_{2}$ inhibited root hair polar expansion, whereas treatment with ROS scavengers (e.g., ascorbic acid) caused root hair bursting (16), reinforcing the notion that apo ROS modulates cell growth by impacting cell-wall properties. In this manner, apo ROS molecules $\left({ }_{\text {apo }} \mathrm{H}_{2} \mathrm{O}_{2}\right)$ coupled to PER activity directly affect the degree of cell-wall crosslinking $(14,20)$ by oxidizing cell-wall compounds and leading to rigidification of the wall in peroxidative cycles (16). The identified PER1,44,60,73 directly contribute to PER activity in peroxidative cycles. On the other hand, apo ROS coupled to PER activity enhances nonenzymatic wall loosening by producing oxygen radical species (e.g., $\bullet \mathrm{OH})$ and promoting polar growth in hydroxylic cycles (33). It is unclear how opposite effects on cell-wall polymers are coordinated during polar growth $(20,33)$. Finally, PERs also contribute to the superoxide radical $\left(\mathrm{O}_{2}{ }^{\bullet-}\right)$ pool by oxidizing singlet oxygen in the oxidative cycle, thereby affecting ${ }_{\text {apo }} \mathrm{H}_{2} \mathrm{O}_{2}$ levels. Although ROS is produced in the apoplast, influencing cellwall properties during cell expansion, $\mathrm{H}_{2} \mathrm{O}_{2}$ is also present at high levels in the cytoplasm during polar growth (17). In agreement, $\mathrm{H}_{2} \mathrm{O}_{2}$ influx from the apoplast was demonstrated for several plant plasma membrane intrinsic proteins (PIPs) in heterologous systems $(34,35)$ as well as in plant cells $(36)$. Because cytosolic acidification triggers a drastic reduction in the water permeability of several PIPs, it is plausible that oscillating $\mathrm{pH}$ changes in 
growing root hairs (17) directly regulate the cycles of ${ }_{c y t} \mathrm{H}_{2} \mathrm{O}_{2}$ influx. Once $\mathrm{H}_{2} \mathrm{O}_{2}$ molecules are transported into the cytoplasm, they trigger downstream responses linked to polar growth $(12,18)$.

\section{Materials and Methods}

Root Hair Phenotype. For quantitative analysis of root hair phenotypes in rboh mutants and WT Col-0, 200 fully elongated root hairs were measured $(n=$ 30 roots) from seedlings grown on vertical plates for $10 \mathrm{~d}$. Values are reported as the mean \pm SD using ImageJ $1.50 \mathrm{~b}$ software $(\mathrm{NIH})$. Measurements made on images were captured with an Olympus SZX7 zoom microscope equipped with a Q-Color5 digital camera. Ten-day-old roots for $\mathrm{ARF}^{\mathrm{OE}}$ were digitally photographed with a stereomicroscope (M205 FA; Leica) at a $40 \times$ magnification. The hair length of 8 to 10 consecutive hairs protruded perpendicularly from each side of the root, for a total of 16 to 20 hairs from both sides of the root.

$\mathrm{H}_{2}$ DCF-DA Probe Used to Measure Total ROS. Growth of Arabidopsis seeds on a plate was done with $1 \%$ sterile agar for $8 \mathrm{~d}$ in a chamber at $25{ }^{\circ} \mathrm{C}$ with continuous light. These seedlings were incubated in darkness on a slide for 10 min with $50 \mu \mathrm{M} \mathrm{2}$ 2 $^{\prime} 7^{\prime}$-dichlorodihydrofluorescein diacetate at room temperature. Samples were observed with a confocal microscope equipped with a 488-nm argon laser and BA510IF filter sets. A 10× objective was used, 0.30 N.A., 4.7 laser intensity, 1.1 offset, 440 photomultiplier (PMT) (for highest ROS levels), 480 PMT (for ROS media), and gain 3. Images were taken scanning XZY with $2 \mu \mathrm{m}$ between focal planes. Images were analyzed using ImageJ. To measure ROS highest levels, a circular region of interest (ROI) $(r=$ 2.5) was chosen in the zone of the root hair with the highest intensities. To

1. Menand B, et al. (2007) An ancient mechanism controls the development of cells with a rooting function in land plants. Science 316:1477-1480.

2. Datta S, Prescott H, Dolan L (2015) Intensity of a pulse of RSL4 transcription factor synthesis determines Arabidopsis root hair cell size. Nat Plants 1:15138.

3. Slabaugh E, Held M, Brandizzi F (2011) Control of root hair development in Arabidopsis thaliana by an endoplasmic reticulum anchored member of the R2R3-MYB transcription factor family. Plant $J$ 67:395-405.

4. Xu P, et al. (2014) HDG11 upregulates cell-wall-loosening protein genes to promote root elongation in Arabidopsis. J Exp Bot 65:4285-4295.

5. Yi K, Menand B, Bell E, Dolan L (2010) A basic helix-loop-helix transcription factor controls cell growth and size in root hairs. Nat Genet 42:264-267.

6. Salazar-Henao JE, Schmidt W (2016) An inventory of nutrient-responsive genes in Arabidopsis root hairs. Front Plant Sci 7:237.

7. Song L, et al. (2016) The molecular mechanism of ethylene-mediated root hair development induced by phosphate starvation. PLoS Genet 12:e1006194.

8. Guilfoyle T, Hagen G, Ulmasov T, Murfett J (1998) How does auxin turn on genes? Plant Physiol 118:341-347.

9. Fukaki H, Tameda S, Masuda H, Tasaka M (2002) Lateral root formation is blocked by a gain-of-function mutation in the SOLITARY-ROOT/IAA14 gene of Arabidopsis. Plant J 29:153-168.

10. Bargmann BOR, et al. (2013) A map of cell type-specific auxin responses. Mol Syst Biol 9:688.

11. Okushima $Y$, et al. (2005) Functional genomic analysis of the AUXIN RESPONSE FACTOR gene family members in Arabidopsis thaliana: Unique and overlapping functions of ARF7 and ARF19. Plant Cell 17:444-463.

12. Foreman J, et al. (2003) Reactive oxygen species produced by NADPH oxidase regulate plant cell growth. Nature 422:442-446.

13. Wu J, et al. (2010) Spermidine oxidase-derived $\mathrm{H}_{2} \mathrm{O}_{2}$ regulates pollen plasma membrane hyperpolarization-activated $\mathrm{Ca}^{2+}$-permeable channels and pollen tube growth. Plant J 63:1042-1053.

14. Lee Y, Rubio MC, Alassimone J, Geldner N (2013) A mechanism for localized lignin deposition in the endodermis. Cell 153:402-412.

15. Xie H-T, Wan Z-Y, Li S, Zhang Y (2014) Spatiotemporal production of reactive oxygen species by NADPH oxidase is critical for tapetal programmed cell death and pollen development in Arabidopsis. Plant Cell 26:2007-2023.

16. Orman-Ligeza B, et al. (2016) RBOH-mediated ROS production facilitates lateral root emergence in Arabidopsis. Development 143:3328-3339.

17. Monshausen GB, Bibikova TN, Messerli MA, Shi C, Gilroy S (2007) Oscillations in extracellular $\mathrm{pH}$ and reactive oxygen species modulate tip growth of Arabidopsis root hairs. Proc Natl Acad Sci USA 104:20996-21001.

18. Takeda S, et al. (2008) Local positive feedback regulation determines cell shape in root hair cells. Science 319:1241-1244.

19. Boisson-Dernier A, et al. (2013) ANXUR receptor-like kinases coordinate cell wall integrity with growth at the pollen tube tip via NADPH oxidases. PLOS Biol 11:e1001719. measure ROS mean, the total area of the root hair was taken. Pharmacological treatments were carried out with a combination of the following reagents: $100 \mathrm{nM}$ and $5 \mu \mathrm{M}$ IAA, $15 \mu \mathrm{M}$ VAS2870, and $100 \mu \mathrm{M}$ SHAM. In the case of a short time experiment with IAA, 8-d-old WT plants were incubated with $100 \mathrm{nM}$ IAA for $3 \mathrm{~min}$ and coincubated for $7 \mathrm{~min}$ with $100 \mathrm{nM}$ IAA and $50 \mu \mathrm{M} \mathrm{H}_{2}$ DCF-DA at room temperature. We washed the sample with MS $0.5 \times$ solution, and image acquisition was done with a $10 \times$ objective and $400 \mathrm{~ms}$ of exposure time on an epifluorescence microscope (Imager A2; Zeiss). As a control, we incubated 10 -d-old WT plants with MS $0.5 \times$ solution for 3 min and coincubated for $7 \mathrm{~min}$ with $\mathrm{MS} 0.5 \times$ and $50 \mu \mathrm{M} \mathrm{H_{2 } D C F - D A}$ at room temperature. To measure ROS levels, a circular ROI $(r=2.5)$ was taken at the tip of the root hair. Values are reported as the mean \pm SD using ImageJ $1.50 \mathrm{~b}$ software.

ACKNOWLEDGMENTS. We thank T. Beeckman, A. Costa, L. Cárdenas, I. De Smet, L. Dolan, N. Geldner, U. Grossniklaus, T. Hamann, J.-Y. Kim, J. KleineVehn, and D. Weijers for materials; and the H.-T.C. and J.M.E. laboratories for discussions. We thank A. R. Kornblihtt for advice and an anonymous editor for English copy edition. We thank the Arabidopsis Biological Resource Center (ABRC) (Ohio State University) for providing T-DNA seed lines. J.P.M., A.D.N., and J.M.E. are investigators of the National Research Council (CONICET), Argentina. This work was supported by grants from ANPCyT (PICT2013-003, PICT2014-504, and ICGEB2016; to J.M.E.) and the MidCareer Researcher Program (2015002633) of the National Research Foundation and the Next-Generation BioGreen 21 Program (Agricultural Genome Center PJ011195) of the Rural Development Administration (to H.-T.C.). H.S.C. was partially supported by the Stadelmann-Lee Scholarship, Seoul National University.

20. Passardi F, Penel C, Dunand C (2004) Performing the paradoxical: How plant peroxidases modify the cell wall. Trends Plant Sci 9:534-540.

21. Brouwer KS, van Valen T, Day DA, Lambers H (1986) Hydroxamate-stimulated O(2) uptake in roots of Pisum sativum and Zea mays, mediated by a peroxidase: Its consequences for respiration measurements. Plant Physiol 82:236-240.

22. Schlereth $A$, et al. (2010) MONOPTEROS controls embryonic root initiation by regulating a mobile transcription factor. Nature 464:913-916.

23. Galli $M$, et al. (2015) Auxin signaling modules regulate maize inflorescence architecture. Proc Natl Acad Sci USA 112:13372-13377.

24. Lee M-S, An J-H, Cho H-T (2016) Biological and molecular functions of two ear motifs of Arabidopsis IAA7. J Plant Biol 59:24-32.

25. Tsukagoshi H, Busch W, Benfey PN (2010) Transcriptional regulation of ROS controls transition from proliferation to differentiation in the root. Cell 143:606-616.

26. Lu D, Wang T, Persson S, Mueller-Roeber B, Schippers JHM (2014) Transcriptional control of ROS homeostasis by KUODA1 regulates cell expansion during leaf development. Nat Commun 5:3767.

27. Vijayakumar P, Datta S, Dolan L (2016) ROOT HAIR DEFECTIVE SIX-LIKE4 (RSL4) promotes root hair elongation by transcriptionally regulating the expression of genes required for cell growth. New Phytol 212:944-953.

28. Hwang Y, Choi HS, Cho HM, Cho HT (2017) Tracheophytes contain conserved orthologs of a basic helix-loop-helix transcription factor that modulate ROOT HAIR SPECIFIC genes. Plant Cell 29:39-53.

29. Sundaravelpandian K, Chandrika NN, Schmidt W (2013) PFT1, a transcriptional Mediator complex subunit, controls root hair differentiation through reactive oxygen species (ROS) distribution in Arabidopsis. New Phytol 197:151-161.

30. Yun BW, et al. (2011) S-nitrosylation of NADPH oxidase regulates cell death in plant immunity. Nature 478:264-268.

31. Nestler J, et al. (2014) Roothairless5, which functions in maize (Zea mays L.) root hair initiation and elongation encodes a monocot-specific NADPH oxidase. Plant $J$ 79:729-740

32. Kwon T, et al. (2015) Transcriptional response of Arabidopsis seedlings during spaceflight reveals peroxidase and cell wall remodeling genes associated with root hair development. Am J Bot 102:21-35.

33. Dunand $C$, Crèvecoeur $M$, Penel $C$ (2007) Distribution of superoxide and hydrogen peroxide in Arabidopsis root and their influence on root development: Possible interaction with peroxidases. New Phytol 174:332-341.

34. Dynowski M, Schaaf G, Loque D, Moran O, Ludewig U (2008) Plant plasma membrane water channels conduct the signalling molecule $\mathrm{H}_{2} \mathrm{O}_{2}$. Biochem J 414:53-61.

35. Miller EW, Dickinson BC, Chang CJ (2010) Aquaporin-3 mediates hydrogen peroxide uptake to regulate downstream intracellular signaling. Proc Natl Acad Sci USA 107:15681-15686.

36. Tian S, et al. (2016) Plant aquaporin AtPIP1;4 links apoplastic $\mathrm{H}_{2} \mathrm{O}_{2}$ induction to disease immunity pathways. Plant Physiol 171:1635-1650. 